\title{
Lipid Autacoid Medicine: A New Fundament for Pain Medicine
}

\section{Jan M Keppel Hesselink}

Institute for Neuropathic Pain, the Netherlands

*Corresponding author: Jan M Keppel Hesselink, Institute for Neuropathic Pain, the Netherlands, Tel: +31306939022; E-mail: jan@neuropathie.nu

Rec date: Feb 03, 2018; Acc date: Feb 07, 2018; Pub date: Feb 13, 2018

Copyright: (c) 2018 Hesselink JMK, this is an open-access article distributed under the terms of the Creative Commons Attribution License, which permits unrestricted use, distribution, and reproduction in any medium, provided the original author and source are credited.

\section{Letter to Editor}

Autacoid medicine is based on formulations containing autacoids or autacoid derivatives, wherein autacoids are 'local tissue hormones' or modulating factors, which affect the function of nearby cells and/or tissues, and are subsequently metabolized by the same tissues [1]. Some neurotransmitters such as NO, ATP and a number of endocannabinoids such as 2-Arachidonoylglycerol (2-AG) can also be classified as autacoids. The word autacoid originates from the Greek autos, meaning self and akos, meaning a drug. Thus, autacoids are 'selfhealing' molecules.

As early as 1981, it was recognized that autacoids can be regarded as mediators of a variety of immune functions, and that the concentration of autacoids in tissues during inflammation is sufficient to allow them to attract a number of immune cells to the site of inflammation, thereby regulating both cell-mediated and humoral immunity [2]. Most of these autacoids play a key role in chronic pain states. For a long time, it has been acknowledged that these "self-medications" can function as an alternative to a number of drugs.

Autacoid medicine builds upon the innate immune mechanisms of defense and repair. Autacoid therapy offers a number of advantages over the New Chemical Entity (NCE) based therapies, the most prominent of them being lack of associated toxic metabolites by virtue of these molecules being endogenous. Therefore, long-term safety issues are ruled out.

Lipid autacoids are perhaps the most well studied of all autacoids. These autacoids and their derivatives create a healthy balance in case of overactive inflammation, and aid in the resolution of the inflammation and the subsequent wound healing. This feature is one of the key aspects of lipid autacoid role in neuropathic pain. The lipid autacoid family is very diverse, and varies from small molecular-weight signaling lipids to high-molecular-weight glycerophospholipids, in addition to their many structural isomers. This makes profiling of these molecules very difficult.

Alessandro Bruni from the Department of Pharmacology, University of Padova in 1988 was perhaps the first to identify that lipid autacoids were synthesized in the plasma [3]. Palmitoylethanolamide (PEA) is one of the most crucial members of the lipid autacoid family. It was identified by the Italian Nobel laureate Rita Levi-Montalcini in the 1990s [4]. She was the first to highlight the (neuro-) repair function of autacoids.

The first patents on lipid autacoids were filed by a small Italian company, Life Group Spa in the 1990s. In these patents the research of Levi-Montalcini on PEA its derivatives in inflammatory models can be found [5]. With more than 650 PubMed indexed papers, PEA is by far the most explored lipid autacoid in both preclinical as well as clinical studies. There are many evidences that the anti-inflammatory effects of PEA can be used to treat a wide variety of disorders such as:
Alzheimer's disease, arthritis, asthma, depression, glaucoma, multiple sclerosis, reperfusion injury, sepsis, and sciatic and neuropathic pain. The neuroprotective profile of PEA revealed that it possesses retinoprotective properties [6].

In the 1980s, PEA was first registered as a drug for the treatment of respiratory viral indications in Spain (brand name Palmidrol). Subsequently, new neutraceutical formulations of PEA were developed and it became more widely available. It is important to highlight that although PEA is classified currently as a supplement in Europe, its therapeutic effects have been well-documented in a number of placebo-controlled randomized clinical trials. Furthermore, there is sufficient clinical evidence to the effect that the use of PEA as a supplement is safe.

The analgesic and anti-inflammatory effects of PEA have also been documented. In humans, PEA doses of up to $100 \mathrm{mg} / \mathrm{kg}$ body weight have been administered without any apparent adverse effects. Currently, there is substantial clinical data available on PEA [6-12]. PEA use appears to be safe and effective in a wide range of neuropathic pain syndromes, ranging from diabetic neuropathy to chronic idiopathic axonal neuropathy. In nerve entrapment neuropathy syndromes such as carpal tunnel syndrome and sciatic pain, a number of placebo controlled clinical studies support the effective and safe use of PEA. Analysis of data from a randomized controlled trial involving over 600 sciatic pain patients revealed an NNT (Number needed to treat) of less than 2 [12]. Therefore, pain physicians should give due consideration to the use of PEA in humans.

A number of formulations of PEA are available in the market, but only the formulations containing micro PEA have been documented to be safe and effective. However, only three of these formulations are present in neutraceutical supplements: optimized PEA (PEA-opt), micronized PEA (PEA-m), and ultra-micronized PEA (PEA-um).

We have been working with PEA in our clinic for the past 8 years, and have treated many patients suffering from axonal neuropathies such as chronic idiopathic axonal neuropathy (CIAP), diabetic neuropathy, and sciatic pain $[8,12]$. According to our studies, a daily dose of $1200 \mathrm{mg}$ for at least two months can be safely recommended in neuropathic pain. It seems that PEA resets the system in the intermittent period. Our findings also reveal that, following a doubling of this dose, partial responders can be transformed into full responders.

Therefore, Lipid autacoids represent a bright ray of hope for pain medicine especially as their side-effects are benign, and as they are metabolized using endogenous metabolic pathways; there are no surprises as to their primary and secondary metabolites. Serious Research and Development in this field would open the door to safer and much more effective pain medication. Now that a number of patents pertaining to autacoids have expired, new possibilities have opened-up for generic formulations of autacoids to enter the market. 
Citation: Hesselink JMK (2018) Lipid Autacoid Medicine: A New Fundament for Pain Medicine. Gen Med (Los Angeles) 6: e115. doi:

\section{Acknowledgement}

No funding or sponsorship was received for the publication of this article.

\section{Disclosure}

Jan M Keppel Hesselink declares that he has no conflict of interest.

\section{References}

1. Keppel Hesselink JM (2015) The terms 'autacoid', 'hormone' and 'chalone'and how they have shifted with time. Auton Autacoid Pharmacol 35: 51-58.

2. Melmon KL, Rocklin RE, Rosenkranz RP (1981) Autacoids as modulators of the inflammatory and immune response. Am J Med 71: 100-106.

3. Bruni A (1988) Autacoids from membrane phospholipids. Pharmacol Res Commun 20: 529-544.

4. Levi-Montalcini R, Skaper SD, Dal Toso R, Petrelli L, Leon A, et al. (1996) Nerve growth factor: From neurotrophin to neurokine. Trends Neurosci 19: 514-520.

5. https://www.google.ch/patents/US5506224
6. Keppel Hesselink JM, Costagliola C, Fakhry J, Kopsky DJ (2015) Palmitoylethanolamide, a natural retinoprotectant: Its putative relevance for the treatment of glaucoma and diabetic retinopathy. J Ophthalmol 2015: 1-9.

7. Hesselink JM, Kopsky DJ (2015) Palmitoylethanolamide, a neutraceutical, in nerve compression syndromes: Efficacy and safety in sciatic pain and carpal tunnel syndrome. J Pain Res 8: 729.

8. Hesselink JK (2013) Chronic idiopathic axonal neuropathy and pain, treated with the endogenous lipid mediator palmitoylethanolamide: A case collection. Int Med Case Rep J 6: 49.

9. Keppel Hesselink JM (2012) New targets in pain, non-neuronal cells, and the role of palmitoylethanolamide. Open Pain J 5: 12-23.

10. Keppel Hesselink JM, Hekker TA (2012) Therapeutic utility of palmitoylethanolamide in the treatment of neuropathic pain associated with various pathological conditions: A case series. J of Pain Res 5: 437442 .

11. Keppel Hesselink JM (2013) Chronic idiopathic axonal neuropathy and pain, treated with the endogenous lipid mediator palmitoylethanolamide: A case collection. Int Med Case Rep J 6: 49-53.

12. Keppel Hesselink JM, DJ Kopsky (2015) Palmitoylethanolamide, a nutraceutical, in nerve compression syndromes: Efficacy and safety in sciatic pain and carpal tunnel syndrome. J Pain Res 8: 729-734. 